\title{
LA FALACIA DE LA EDUCACIÓN INTERCULTURAL: UN ANÁLISIS DE LA LEGISLACIÓN EDUCATIVA, CULTURAL Y RELACIONES DE PODER POLÍTICO EN BOLIVIA*
}

\author{
THE FALLACY OF INTERCULTURAL EDUCATION: \\ AN ANALYSIS OF EDUCATIONAL AND CULTURAL LEGISLATION AND \\ POLITICAL POWER RELATIONS IN BOLIVIA
}

Claudia Vaca Flores**

\section{Resumen}

En los últimos 15 años, Bolivia ha experimentado diversos cambios a nivel político, educativo y cultural, expresados en la promulgación y ejecución de nuevas leyes que reconfiguraron las relaciones de poder en la sociedad boliviana, impactando en la educación y en las interacciones de la diversidad cultural que coexisten en Bolivia desde antes de la colonia española. El presente artículo tiene como objetivo analizar las relaciones de poder en torno a la interculturalidad educativa y las políticas culturales y de lectura en Bolivia. Se aplicó el método de análisis documental y entrevista semiestructurada a profesores de la federación rural y urbana de maestros, en las ciudades de Cochabamba y Santa Cruz de la Sierra. Entre los resultados de la investigación se devela el objetivo político indigenista de lo intercultural en la ley de educación, persiste la imagen andino céntrica de Bolivia, se repite el patrón de la colonización a nivel interétnico, se debilita el alcance técnico-económico y pedagógico de las direcciones departamentales de educación; a nivel de actualización docente, se impone la dogmatización

\footnotetext{
* La redacción de este artículo ha sido posible gracias a la beca docente del Doctorado en educación de la Universidad Católica de la Santísima Concepción, Chile.

** Doctoranda en Educación. Universidad Católica de la Santísima Concepción. email: cvaca@doctoradoedu.ucsc.cl, ORCID https://orcid.org/0000-0001-7237-5715
} 
político-ideológica en menoscabo de la actualización pedagógica, didáctica, tecnológica y de diversidad cultural.

\title{
Palabras clave:
}

Legislación, educación, diversidad cultural, lectura, poder político, profesor.

\begin{abstract}
:
In the last 15 years, Bolivia has experienced various changes at a political, educational and cultural level reflected in the enactment and execution of new laws that reconfigured power relations in Bolivian society, impacting on education and the interactions of the cultural diversity that coexist in Bolivia since before the Spanish colony. In this sense, the present article aims to analyse the power relations around educational interculturality, cultural and reading policies in Bolivia. The method of documentary analysis and semi-structured interview is applied to teachers from the rural and urban federation of teachers in the cities of Cochabamba and Santa Cruz de la Sierra. Among the results of the research, the indigenous political objective of the interculturality in the education law is revealed, the Andean centric image of Bolivia persists, the pattern of colonization at the inter-ethnic level is repeated, the technical-economic and pedagogical scope of the Department of education declines; at the level of teaching updating, political-ideological dogmatization is imposed to the detriment of pedagogical, didactic, technological and cultural diversity update.
\end{abstract}

\section{Keywords:}

Legislation, education, cultural diversity, reading, political power, teacher.

\section{Introducción}

Desde el año 2006 se instala un nuevo gobierno en Bolivia, que establecerá una serie de leyes para respaldar el autoritarismo, la colonización interétnica y la monopolización del poder a largo plazo, bajo categorías conceptuales vigentes a nivel global, como la interculturalidad.

Sucedieron desde ese momento hasta finales del 2019, una serie 
de abusos de poder en contra de las minorías étnicas de tierras bajas, desde una lógica arcaica de revanchismo histórico que normaliza la violencia estructural preexistente en Bolivia, y se posicionarán dinámicas de trabajo en el sistema educativo que invisibilizan sistemáticamente la diversidad cultural de Bolivia a nivel global.

En este contexto, el presente artículo tiene el objetivo de analizar las relaciones de poder gestadas en torno a la interculturalidad educativa en Bolivia a partir de la ejecución de 4 leyes, su impacto en la realidad educativa del profesorado, las relaciones de poder y las relaciones culturales diversas que coexisten en todo el territorio boliviano. Dichas leyes son, la Constitución Política del Estado Plurinacional de Bolivia (2009), la Ley de Educación 070 "Avelino Siñani-Elizardo Pérez" (2010), la Ley 366 del Libro y la Lectura "Oscar Alfaro" (2013) y la Ley Marco de Autonomías “Andrés Ibáñez” (2010), creadas entre la primera y segunda gestión del estado plurinacional de Bolivia.

El punto de partida para dicho análisis es la siguiente interrogante: ¿Cuál es la noción de interculturalidad educativa y el valor que asigna el estado plurinacional de Bolivia a la lectura, la memoria oral, las bibliotecas y la educación? Luego de revisar los capítulos y artículos de cada ley, se analizó con profundidad la Ley 366 del Libro y la Lectura y la Ley 070 de Educación; de allí proviene el análisis de categorías y unidades que permiten conocer el ecosistema educativo de la lectura y lo intercultural en Bolivia.

\section{Método:}

El estudio se desarrolló desde una perspectiva cualitativa, con enfoque hermenéutico y análisis crítico del discurso en torno a la interculturalidad educativa y los argumentos prevalecientes en los 8 profesores entrevistados. Se realizó un análisis documental de la legislación boliviana generada en el gobierno del Movimiento al Socialismo (MAS), en torno a los constructos de: literacidad, oralidad, interculturalidad y educación; referidos en los mismos documentos. Las entrevistas semiestructuradas se realizaron a partir de estos constructos. Seguidamente se efectuó un análisis corpus 
de frecuencia de palabras en los documentos y en las entrevistas a profesores, a través del software NVivo. A continuación, un detalle del procedimiento desarrollado.

Tabla 1. Las leyes analizadas y la política educativa intercultural

\begin{tabular}{|c|c|c|}
\hline Nombre & Tipo & Fuente \\
\hline $\begin{array}{l}\text { Constitución Política del } \\
\text { Estado Plurinacional de } \\
\text { Bolivia }\end{array}$ & Constitución & $\begin{array}{l}\text { Asamblea Legislativa del Estado } \\
\text { Plurinacional de Bolivia / } \\
\text { Gaceta oficial del Estado }\end{array}$ \\
\hline $\begin{array}{l}\text { Ley de educación del Estado } \\
\text { Plurinacional de Bolivia, } \\
\text { Avelino Siñani (2009-2010) }\end{array}$ & Ley & $\begin{array}{l}\text { Asamblea Legislativa del Estado } \\
\text { Plurinacional de Bolivia / } \\
\text { Gaceta oficial del Estado }\end{array}$ \\
\hline $\begin{array}{l}\text { Ley marco de } \\
\text { autonomías indígenas y } \\
\text { departamentales (2009) }\end{array}$ & Ley & $\begin{array}{l}\text { Asamblea Legislativa del Estado } \\
\text { Plurinacional de Bolivia / } \\
\text { Gaceta oficial del Estado }\end{array}$ \\
\hline $\begin{array}{l}\text { Ley del libro y la lectura, } \\
\text { Oscar Alfaro (2013) }\end{array}$ & Ley & $\begin{array}{l}\text { Asamblea Legislativa del Estado } \\
\text { Plurinacional de Bolivia / } \\
\text { Gaceta oficial del Estado }\end{array}$ \\
\hline $\begin{array}{l}\text { Bono escolar de } \\
\text { transferencia condicionada } \\
\text { Juancito Pinto y Programa } \\
\text { de Alfabetización "Yo sí } \\
\text { puedo." }\end{array}$ & $\begin{array}{l}\text { Programa } \\
\text { enmarcado en } \\
\text { la Ley } 070 \text { de } \\
\text { educación }\end{array}$ & $\begin{array}{l}\text { Asamblea Legislativa del Estado } \\
\text { Plurinacional de Bolivia / Gace- } \\
\text { ta oficial del Estado/ Ministerio } \\
\text { de Educación de Bolivia }\end{array}$ \\
\hline
\end{tabular}

Fuente: Elaboración propia (abril, 2019)

En torno a estas leyes se establecieron nociones conceptuales y políticas de los constructos: interculturalidad, lectura, memoria oral y educación. Se generó una lista de significados de estos constructos en el discurso de los profesores entrevistados para identificar los campos semánticos, congruencias o incongruencias internas en los documentos, entre el discurso de los profesores y los objetivos implícitos de la legislación vigente.

Desde allí se analizaron las relaciones de poder en torno a las políticas educativas y culturales, la agudización del centralismo político-ideológico de la educación en Bolivia y la finalidad de estas leyes a nivel estructural en las políticas culturales y educativas del país. 
El análisis de los 4 documentos y las entrevista a profesores se realizó a partir de categorías y subcategorías, para generar categorías emergentes en el ámbito de la administración educativa, cultural, políticas sindicales y departamentales. Todas estas categorías se analizaron a partir de la siguiente tabla de análisis de contenido temático:

Tabla 2. Análisis de contenido temático:

\section{Categorías de Análisis}

\begin{tabular}{ll}
\hline Categoría 1. & $\begin{array}{l}\text { Interculturalidad educativa y políticas de lectura en } \\
\text { Bolivia, desde la descentralización administrativa en } \\
\text { los últimos 15 años. }\end{array}$ \\
Subcategoría 1.1. & $\begin{array}{l}\text { Actualización bibliográfica y sistema nacional de bi- } \\
\text { bliotecas escolares con presupuestos departamentales } \\
\text { y municipales para la contratación de mediadores } \\
\text { y animadores de lectura en las bibliotecas públicas } \\
\text { municipales y campesinas. }\end{array}$ \\
Subcategoría 1.2. & $\begin{array}{l}\text { Construcción de bibliotecas públicas y unidades edu- } \\
\text { cativas públicas con bibliotecas en las principales } \\
\text { ciudades de Santa Cruz de la Sierra. }\end{array}$ \\
\hline Categoría 2. & $\begin{array}{l}\text { Ecosistema de la educación intercultural y lectura en } \\
\text { Bolivia desde la Ley de Descentralización y Autono- } \\
\text { mías. }\end{array}$ \\
Subcategoría 2.1. & $\begin{array}{l}\text { Competencias y recursos asignados para lectura y } \\
\text { educación, patrimonio cultural inmaterial en Bolivia. }\end{array}$ \\
\hline Categoría 3. & Educación intercultural \\
Subcategoría 3.1. & Lectura e interculturalidad \\
\hline
\end{tabular}

Fuente: Elaboración propia (mayo, 2019)

En relación al establecimiento de la calidad científica de las categorías, se siguió el criterio de confirmabilidad (Arias y Giraldo, 2011), el corpus textual reunió 4 leyes bolivianas de carácter constitucional, que dialogan con dos declaraciones de la UNESCO (2003 y 2005) en relación con la educación y la lectura, una declaración del Centro Regional del Libro y la Lectura para América Latina y el 
Caribe (Cerlalc) referente al compromiso con la lectura de parte de los Estados (2020). Se analizó todo ello desde la orientación hermenéutico-interpretativa. A continuación, el detalle de la rigurosidad científica aplicada en cada categoría de análisis.

Tabla 3. Detalle de la rigurosidad científica aplicada en cada categoría de análisis

\section{Calidad científica}

Categoría 1: $\quad$ Desde el Cerlalc y distintas estadísticas generadas para medir los niveles de lectura, memoria oral y niveles de inversión de los gobiernos en este tema, se han establecido las categorías elegidas y contextualizadas en Bolivia: Políticas de lectura en Bolivia y los aportes de Claudia Vaca en el Ethos Lector (2018).

Desde la ley marco de autonomías estudiada por el Dr. Juan Carlos Urenda (2018), Dr. Esteban Valenzuela Van Treek (2017) y Carlos Hugo Molina (2018), se establece la categoría de descentralización administrativa desde el ámbito de territorialidad y cantidad de recursos económicos asignados para determinado tema de interés público, en este caso la lectura.

La pertinencia científica de esta categoría tiene sus bases en los estudios sobre políticas de patrimonio cultural inmaterial (UNESCO, 2005), políticas públicas de lectura en Latinoamérica, declaraciones internacionales establecidas por la UNESCO y el PNUD (ODS 2030, Declaración INCHEON 2015), el marco legal nacional e internacional de la misma ley del libro y la lectura, la ley de educación 070 de Bolivia.

Subcategoría 1.1. En ambas subcategorías referidas a la construcción de bibliotecas y actualización bibliográfica, el Cerlalc, como organismo de la UNESCO (2015) establece el carácter obligatorio de los gobiernos para atender las necesidades de fomento a la lectura en los países, desde la construcción de bibliotecas públicas, escolares y actualización bibliográfica.

Categoría 2: $\quad$ Se analiza transversalmente la Ley Marco de Autonomías de Bolivia, con énfasis en las competencias y niveles de administración, desde allí se devela la ausencia de recursos y competencias en el ámbito educativo y cultural. 
Se toman los aportes en los procesos de descentralización administrativa y política de autores como: Paula Peña (2020), Esteban Valenzuela y Claudia Vaca (2020) y Juan Carlos Urenda (2018).

La pertinencia científica de esta categoría tiene sus bases en la ciencia política: eje de políticas educativas y culturales.

Subcategoría 2.1. En las competencias y recursos asignados para la lectura y educación se toman los aportes del Dr. Juan Carlos

Subcategoría 2.2. Urenda (2018), investigador experto en descentralización en Bolivia, así como los aportes de Valenzuela Van Treek en relación a las ciudades contrapeso político y cultural, Vaca (2019) y Ábrego y Quiróz (2020) en temas de educación intercultural, gestión cultural, bibliotecas populares y lectura en Bolivia.

Fuente: Elaboración propia (entre junio 2019 y agosto 2020)

Las categorías iniciales de análisis, las subcategorías y categorías emergentes de las entrevistas a profesores, se interpretaron a partir de esta matriz:

\section{Grilla o matriz de análisis documental y de corpus de las entrevistas}

\begin{tabular}{|c|c|c|}
\hline $\begin{array}{l}\text { Categorías } \\
\text { de análisis }\end{array}$ & Unidades & Interpretación \\
\hline $\begin{array}{l}\text { Categoría } 1 . \\
\text { Subcategoría 1.1. } \\
\text { Subcategoría 1.2. }\end{array}$ & $\begin{array}{l}\text { a) Las políticas de } \\
\text { lectura en Bolivia. } \\
\text { b) Ecosistema del } \\
\text { libro y la lectura } \\
\text { en Bolivia: Plan } \\
\text { Nacional de } \\
\text { lectura y sistema } \\
\text { nacional de } \\
\text { bibliotecas } \\
\text { escolares. }\end{array}$ & $\begin{array}{l}\text { En su artículo } 1 \text { la ley del libro y la } \\
\text { lectura "Oscar Alfaro" se enfatiza } \\
\text { el objetivo de la ley de promover el } \\
\text { ejercicio del derecho a la lectura y } \\
\text { escritura como instrumentos para la } \\
\text { educación, en condiciones de liber- } \\
\text { tad, equidad social y respeto a la di- } \\
\text { versidad de expresiones culturales, } \\
\text { generando políticas públicas, planes } \\
\text { y acciones de fomento a la escritura, } \\
\text { lectura y acceso al libro, la creación } \\
\text { cultural, literaria, académica y cien- } \\
\text { tífica. } \\
\text { Se resalta el carácter enunciativo de } \\
\text { la diversidad cultural del libro y la lec- } \\
\text { tura, es decir su alcance en distintas }\end{array}$ \\
\hline
\end{tabular}


lenguas y formatos para que llegue a todo el territorio boliviano, cuya cualidad única en el mundo es la diversidad cultural viva en sus lenguas y hablantes, por lo cual se explicita la participación de todos en la lectura, esto último tiene relación directa con la Ley de Educación Boliviana 070, que en el capítulo II, artículo 2 dice: La educación es comunitaria, democrática, participativa y de consensos en la toma de decisiones sobre políticas educativas, reafirmando la unidad en la diversidad y acceso a las herramientas para educarse, como ser el libro y las tecnologías.

Vemos que, en ambos casos de la ley, se explicita el carácter relevante del acceso al libro, la lectura, la participación ciudadana para toma de decisiones en políticas públicas. Pero no se ejecutan ninguno de estos capítulos, como veremos en el análisis.

Categoría 2.

Subcategoría 2.1. intercultural y Subcategoría 2.2. lectura en el sistema político y administrativo de Bolivia

a) Los niveles de administración de las políticas de lectura y educación

b) Los niveles de descentralización
Referente a las políticas públicas de lectura en los 4 niveles de gobierno: nacional, departamental, municipal y originario campesino, se utilizará el término "ecosistema de la lectura en Bolivia”. En este sentido, aquí se dialoga entre los artículos 1 de la ley del libro y la lectura (disposiciones generales), el artículo 2 (puntos 1 , $3,6,7,8)$, al capítulo III referente al plan nacional de lectura, al artículo 11 del capítulo IV, referente a la implementación de bibliotecas y al capítulo VI, referente al carácter administrativo de los comités autónomos y departamentales de lectura. Estos capítulos dialogan con los principios de transparencia y transferencia de competencias de la Ley Marco de Autonomías, donde se enfatiza la participación ciudadana, no se menciona la transferencia de recursos para 
políticas educativas, mucho menos de lectura; no es una prioridad el eje de educación en ninguno de sus niveles de gobierno ni de competencias asignadas: la educación y la lectura; se deduce la permanencia del centralismo administrativo en el ámbito educativo y cultural. El régimen de autonomías tiene como fin distribuir las funciones político-administrativas del Estado de manera equilibrada y sostenible en el territorio para la efectiva participación de las ciudadanas y ciudadanos en la toma de decisiones, la profundización de la democracia y la satisfacción de las necesidades colectivas y del desarrollo socioeconómico integral del país. En ningún momento de la ley se menciona la descentralización o asignación de recursos para políticas de lectura en ninguno de los niveles de gobierno (nacional, departamental, municipal y originario campesino).

Cabe explicitar que, a partir de estas categorías emergentes en el análisis fue necesaria una segunda entrevista a los profesores, que permitió consolidar el análisis documental, desde la interculturalidad crítica hacia el análisis hermenéutico de la legislación boliviana en relación a la lectura, la educación intercultural y la administración política territorial (Albó, 2009) en su ley marco de autonomías y la constitución política del Estado.

\section{Resultados}

En términos generales, se observa que, a partir de las categorías iniciales de análisis, las subcategorías y categorías emergentes se devela un ethos colonizador en el Movimiento al Socialismo (MAS), 
que busca "andinizar" Bolivia, debilitando las variantes socioculturales de tierras bajas, se repite el patrón de la colonización criticada por el mismo (MAS) en sus discursos políticos internacionales, y ejercida a nivel interétnico en el país. Desde una dinámica de debilitamiento interétnico, se fragmentan las dirigencias de tierras bajas, se agudizan las fricciones preexistentes en Bolivia entre diversas regiones, se debilita el alcance técnico y económico de las direcciones departamentales de educación, se genera un ambiente de dogmatización político-ideológico hacia los profesores de Bolivia, además se interfiere en el proceso didáctico y pedagógico de los estudiantes, lo que afecta el desarrollo de sus habilidades y competencias educativas para el siglo XXI. Por último, no se generan actualizaciones pedagógica y didácticas, acorde a las demandas globales, en beneficio de los estudiantes y el desarrollo pleno de sus habilidades y talentos.

Asimismo, se generan hallazgos que abren caminos para otros estudios para reconceptualizar la interculturalidad y superar el indigenismo en el que se ha caído, en el caso boliviano y de varios países de América Latina porque además de 36 pueblos originarios hay mestizaje innegable en el territorio boliviano, el mismo nombre del país es resultado del mestizaje que forma parte de nuestra historia. Es necesario trasladar el debate hacia la interpelación de los documentos y políticas indigenistas que menoscaban el sentido relacional y dialógico de la interculturalidad (Albó, 2009) como categoría conceptual en favor de la diversidad cultural y el diálogo, más allá de los revanchismos históricos y fricciones ideológicas.

Entre los hallazgos relevantes de la investigación se devela la invisibilización de las variantes socioculturales de tierras bajas, se agudiza el patrón de la colonización a nivel interétnico y se debilita el alcance técnico-económico y pedagógico de las direcciones departamentales de educación. A nivel de actualización docente, se observa un menoscabo de la actualización pedagógica, didáctica y tecnológica, acorde a las habilidades que requieren los profesores y estudiantes en el siglo XXI, en relación a la diversidad cultural y acceso a las tecnologías de la comunicación. 
a) La lectura y los derechos de la niñez en el estado plurinacional de Bolivia

A partir de la Ley 366 del Libro y la Lectura "Oscar Alfaro" y la Ley de Educación 070 se abordaron aspectos referentes a la interculturalidad y derechos de la niñez, que impactan directamente el ecosistema de la lectura y educación boliviana y evidencian la utilización de la niñez indígena como factor de propaganda política y manipulación de las estadísticas de Bolivia. Esto se sustenta mediante la técnica corpus, análisis de los párrafos y textos centrales referentes a lectura, educación e interculturalidad, dado que el concepto de interculturalidad es la arista central de las leyes y políticas de los últimos 15 años del estado plurinacional de Bolivia.

El análisis de la Ley del Libro y la Lectura, en diálogo con párrafos y artículos de la Ley de Educación 070 y la Ley Marco de Autonomías, permite identificar niveles ambiguos de competencias políticas y administrativas a nivel departamental. No se actualizan los ítems para profesores formados en las escuelas normales de maestros de cada región, se persiste en el trasplante de profesores para andinizar el país, en lugar de facilitar recursos económicos para los profesores, que ya existen en las zonas del oriente boliviano e invertir en el fortalecimiento de las identidades y el diálogo cultural abierto entre la gran diversidad que habita Bolivia.

En el documento de la Ley 366 del Libro y la Lectura "Oscar Alfaro", se explicita que dicha ley se produce porque el estado tiene la obligación de promover el ejercicio del derecho a la lectura y escritura en condiciones de libertad, equidad social y respeto a la diversidad étnica y cultural, lo cual es coherente si enmarcamos la cita con los derechos culturales y derechos humanos de los pueblos originarios, sumando a ello los objetivos de desarrollo sostenible $2030^{1}$ del Programa de las Naciones Unidas para el Desarrollo Humano (PNUD); la Ley del Libro y la Lectura es fundamental para alcanzar

${ }^{1}$ Los Objetivos de Desarrollo Sostenible también conocidos por sus siglas ODS son una iniciativa impulsada por Naciones Unidas para dar continuidad a la agenda de desarrollo tras los Objetivos de Desarrollo del Milenio. 
el objetivo de una educación de calidad. Asimismo, esta obligación está asignada por la Cerlalc ${ }^{2}$, quien en su nueva agenda por el libro y la lectura (Cerlalc, 2013, p. 15), establece los roles del estado como promotor de la lectura, prescriptor y productor de contenidos culturales, facilitador de las infraestructuras socio-tecnológicas y agente regulador y fiscal, tal como lo prescribe la UNESCO.

Todo el documento se produce para demostrar cumplimiento a los mandatos internacionales, pero en las entrevistas a profesores, que fungen de gestores culturales en muchos casos, estos documentos no sirven a las necesidades educativas y culturales de los estudiantes y profesores de Bolivia, porque no se llegan a reglamentar estas leyes, tampoco se establecen programas y proyectos de investigación sobre patrimonio lingüístico, tradición oral, literatura y lectura para las diversas zonas rurales, urbanas y periurbanas de Bolivia.

En palabas del profesor Marcelino:

“...estas leyes únicamente sirven para pagar los salarios de los abogados que las generan y de los intelectuales que están fabricando palabras y discursos para el presidente o los que están a cargo de justificar sus roles políticos. A nosotros no nos llegan libros nuevos, que sirvan para nuestros niños y jóvenes, llegan donaciones de libros sobre testigos de Jehová, o libros viejos de enseñanza de las matemáticas o revistas de belleza, donaciones que hace la gente que no sabe qué hacer con su basura, es triste lo que nos pasa, y cómo el mundo cree que este gobierno ha cambiado nuestra realidad" (Posiva, 2020).

En el caso boliviano, no se han asumido las políticas y compromisos correspondientes, por ello este estudio busca evidenciar y argumentar el estado de la lectura en relación con la educación, la interculturalidad y la pobreza persistente en Bolivia, no por falta de recursos, sino por falta de voluntad política y de priorización en la distribución de los recursos para la lectura y educación. El ecosistema de la lectura está en un proceso de cambio que afecta no solo a

2 El Centro Regional para el Fomento del Libro en América Latina y el Caribe es un organismo intergubernamental bajo los auspicios de la UNESCO, con oficina sede en Colombia. Trabaja en la creación de condiciones para el desarrollo de sociedades lectoras. 
los actores, sino sobre todo al centro del sistema: La educación y sus actores principales en el caso boliviano: la niñez y juventud.

La Ley $\mathrm{N}^{\circ} 366$ del Libro y la Lectura "Oscar Alfaro" emanada de la Asamblea Legislativa del Estado Plurinacional de Bolivia, editada y promulgada por la Gaceta Oficial del Estado el 29 de abril del 2013, se gestó en un contexto de diversas demandas por parte de distintos sectores en torno al libro y la lectura, sintetizados en tres grupos: a) por un lado estaban los representantes del pueblo guaraní, chiquitano, moxeño, quechua y aymará, exigiendo la publicación y generación de material de lectura en sus lenguas originarias, así como la producción audiovisual que les permita rescatar sus tradiciones orales y establecer estudios sistemáticos y participativos de la enseñanza de sus lenguas originarias y transferencia de la memoria oral. Estas demandas se enmarcaban en la Ley de Educación 070 promulgada el 2009; por otro lado, estaban b) los libreros y editores, demandando el impuesto cero para la importación de libros y para la venta de los mismos a la ciudadanía y políticas contra la piratería. Por otra parte, estaban c) los colectivos de escritores independientes, gestores, mediadores, animadores de lectura, educadores y artistas que demandan hasta la actualidad la creación de un plan nacional de lectura como política pública con presupuestos fijos para mediadores de lectura, tanto en las zonas urbanas como rurales, y sobre todo en las zonas más aledañas y periféricas de Bolivia.

En Bolivia también se ha planteado el desafío de crear una cultura de lectura, tanto dentro como fuera de la escuela. En el caso boliviano, la lectura está vinculada a otros procesos que no son únicamente de carácter cognitivo, sino de carácter sociocultural, más aún en las definiciones de educación intercultural y revalorización de los pueblos originarios y campesinos, vertidas en la Ley 070 de Educación en Bolivia (2010). Por eso es importante explorar y elaborar los estudios que se enfocan en analizar la relación entre la tradición oral y la lectoescritura, para generar didácticas contextualizadas, especialmente por su relevancia con la Ley de Educación 070 y sus objetivos de interculturalidad y plurilingüismo; en todo caso las entrevistas y el análisis realizado develan una incongruencia entre 
estas leyes y las decisiones en políticas públicas educativas y culturales en favor de la interculturalidad y el diálogo de saberes.

En la entrevista a la profesora Josefa (2020), se confirma que la producción de leyes no ha logrado la transformación de la realidad política educativa y el diálogo de saberes en Bolivia.

"...nos han dicho que hay que asistir a las capacitaciones de los sábados, porque si no vamos está en riesgo nuestro salario y prevalencia en el sistema educativo, como profesores, nos asignaron como profesores interculturales, pero no hay material de enseñanza de la lengua, entonces uno se tiene que inventar, en realidad hacer lo que siempre hicimos, ser creativos, en parte es bueno, porque transferimos mitos y leyendas locales, lo que si ayudaría mucho es tener herramientas para que los estudiantes a partir de esos mitos, mejoren su comprensión lectora, aprendan más rápido a leer y escribir, necesitamos capacitación técnica en lectura, no es suficiente una ley"

En relación a esta fase del análisis documental y entrevistas vemos que, aunque existe una literatura teórica sobre la lectoescritura como una forma de aprendizaje occidental y la marginación de la tradición oral, hay menos literatura sobre estudios realizados para implementar formas complementarias para la lectoescritura, acorde al desarrollo cognitivo y contexto sociocultural de los pueblos originarios de Bolivia. Lo que falta son estudios sobre cómo organizaciones, escuelas o proyectos en Bolivia están intentando integrar estas dos formas de aprendizaje para transferir su identidad cultural, resguardar la memoria y tradición oral, y establecer el hábito de lectura en los niños y jóvenes.

Como aporte a este análisis, es necesario establecer cómo ha sido teorizada la lectoescritura en relación a la tradición oral. La lectoescritura como una manera de aprender occidental, se ha aceptado, normalizado, e implementado en las escuelas como "universal" y "neutral" sin ser cuestionada. Aún más, la manera en la que se usan los textos culturales para la lectoescritura ha promovido el aprendizaje como una habilidad técnica para desarrollar un pensamiento abstracto, objetivo, lógico, y racional (Zavala 2001). 
Existe una dependencia y creencia absoluta en los libros y textos escolares, es decir, lo que establecen los textos escolares como "datos" y "verdades" no son debatidos, sino aceptados como siendo los únicos "datos" y "verdades" que existen. Además, la información que se encuentra en estos textos y la manera en la que es compartida, es privilegiada sobre los conocimientos y los valores de los estudiantes. Esta dependencia y centralización de una manera de aprender está totalmente en contra de la interculturalidad que se está intentando implementar en Bolivia. Hay discursos y leyes a favor de la interculturalidad y la lectura, se enseña guaraní y quechua de manera obligatoria en el currículo, pero no se asumen políticas públicas a largo plazo y con presupuestos descentralizados para generar material cultural y de lectura que promueva la interculturalidad, el verdadero diálogo de saberes entre las más de 36 identidades lingüísticas con su tradición oral viva, material que podría servir para la mediación de la lectura y mejoramiento del sistema educativo, así como el reconocimiento de la diversidad cultural del país, sin tintes políticos ni fines monopolizantes de una cultura y de una etnia sobre otra.

La imposición del aprendizaje occidental global ha resultado en que otras formas culturales de aprendizaje, como la tradición oral, sean ignoradas y no integradas en los contenidos curriculares y las experiencias educativas de los estudiantes, y en el caso boliviano la enseñanza de únicamente dos variantes culturales del país, es también un modo de monopolizar el currículo y la enseñanza, lo cual se contradice con el diálogo de saberes y el sentido relacional dialógico de la interculturalidad desde niveles más ontológicos y desarrollo del ser. Esta imposición de una manera de aprendizaje sobre otra no sólo se puede discutir en términos de diferencias culturales, sino también en términos de poder. Como expresa en entrevista el profesor, titiritero y gestor sociocultural Zenón (2020): “(...) la lectura y escritura en los pueblos originarios no es sólo un asunto cultural; es además y tal vez sobre todo un hecho de hegemonia, de identidad (...). En este proyecto de homogeneización de lo andino sobre lo amazónico y las tierras bajas, o de exclusión de lo chiquitano, moxeño, tsimané, se persiste en la herramienta de 
subalternización: la escritura de lo ajeno, y se nos quita la posibilidad de lo oral como algo válido para el aprendizaje de la lectura, hay que incorporar lo amazónico en el imaginario de Bolivia a nivel global, y eso se hace desde casa, y en nuestro caso, la ley de educación, la ley del libro y todo lo que produjo el gobierno del MAS no ha servido para integrarnos, todo lo contrario"

Es evidente que existe una conexión entre la tradición escrita y la tradición oral; la conceptualización del alfabetismo y la lectoescritura están siendo desafiadas intelectualmente, particularmente en países como Bolivia, donde se generan instancias políticas que simulan la incorporación del diálogo de saberes y dispersan la atención que pueda tener el país por parte de los referentes internacionales que aportan al desarrollo de políticas culturales.

Al respecto, es relevante hacer una distinción entre saber y conocimiento (Vaca, 2018). Ella es importante, en el presente contexto, para destacar la radical inconmensurabilidad entre ambos. Hay que aclarar que esto no coincide, tal y como indica el profesor Zenón en la entrevista, aunque tiene algunos paralelos, al momento de mirar los atributos culturales y de la memoria oral del saber andino-amazónico al unísono.

Si revisamos también, la distinción propuesta por Vaca (2018) entre episteme y techné, se trata de dos sistemas de conocimiento, distintos y equivalentes, pero complementarios. "Para nosotros, el conocimiento es un resultado directo de la forma en la que nos han educado hasta ahora...", explica el profesor y animador sociocultural Zenón (2020), que se puede entender, en una dimensión fundamental, como un proyecto de conocimiento.

Al respecto, Zenón (2020), en sus actividades de títeres para la lectura trabaja con esta lista diferencial entre conocimiento y saber, al momento de pedir participación de los niños y jóvenes, en relación a las obras que presentan, la mayoría con representaciones de la fauna y flora de las tierras bajas de Bolivia y sus obras las escribe él mismo, desde una estética de fábulas. El ejercicio que les propone es diferenciar conocimiento de saber y obtiene de sus estudiantes lo siguiente: 


\begin{tabular}{ll}
\hline \multicolumn{1}{c}{ Conocimiento } & \multicolumn{1}{c}{ Saber } \\
\hline $\begin{array}{l}\text { Universal, cerebral, analítico } \\
\text { secuencial, impersonal, aplicación } \\
\text { de método organizado, surge de la } \\
\text { mirada técnica y busca la inmedia- }\end{array}$ & $\begin{array}{l}\text { Local-contextual, Sensorial-emocional, } \\
\text { holístico-radial-circular, múltiples } \\
\text { vías de acceso, surge de la crianza, es } \\
\text { vivencial, encuentra en la vida espa- } \\
\text { cios para comprender y reflexionar. }\end{array}$ \\
\hline
\end{tabular}

Esta diferencia no la establece el Ministerio de Educación, ni lo enseñan en sus capacitaciones a profesores. Es decir, la entrevista al profesor y animador sociocultural y de la lectura, Zenón (2020), devela una didáctica propia de su cultura y su experiencia de más de 35 años de trabajo, la cual podría ser reconocida por un estado que verdaderamente valore la educación, la interculturalidad, la lectura y la memoria oral de sus diversos habitantes.

b) Interculturalidad, género y pobreza en el sistema educativo de Bolivia:

Además de los hallazgos en relación al discurso generado desde la producción de leyes que respalden las acciones y accesos a fondos, bajo el argumento de la interculturalidad y el indigenismo, se observa en la realidad del sistema educativo boliviano enormes brechas a nivel de género, accesos a servicios básicos y desarrollo del diálogo intercultural.

Estas tres dimensiones están relacionadas transversalmente con la transmisión de la pobreza entre una y otra generación en los hogares de Bolivia. Según el informe de la CEPAL (2020) el gasto público es 7,3\% del PIB, otro punto que supera el resultado de América Latina, que destina en promedio un 5,4\% del PIB al factor en cuestión. Eso podría explicar la consecuente tasa de alfabetización en personas con 15 años o más que se sitúa en un 95,7\%, comparado con América Latina con un 93,2\% (CEPAL, 20203). Vemos que ha crecido la tasa de alfabetización, pero incluso con el crecimiento

${ }^{3}$ Distribución espacial y urbana de la población de América Latina y el Caribe. Acceso el 6 de febrero. https://bit.ly/3kuOuLv 
de la tasa de alfabetización y escolarización, se registran desafíos a nivel de calidad y cantidad de profesores por estudiantes, cantidad de libros por habitante, especialmente en las zonas rurales donde hay un profesor para toda la comunidad, o en la periferia urbana, donde muchos estudiantes se quedan sin cupo en los establecimientos educativos, sigue el reto de aumentar profesores en secundaria y atender la seguridad laboral y salarial de los profesores recién egresado de las escuelas normales de maestros.

Esto se corrobora en la entrevista a la profesora Eliana (2020):

"Hace más 10 años que vivo en San Ignacio de Moxos, me vine con la ilusión de trabajar como profesora luego de terminar mis estudios, vivi sin salario por 4 años, más bien consegui trabajo en la biblioteca, con el programa de educación alternativa, pero el sueldo es mínimo, y yo sé que tengo derecho a una mejor condición, porque estudié y me preparé para ser profesora, el trámite para que me asignen un item como profesora es muy costoso en lo económico y lo emocional, he decidido sobrevivir con lo que hay y vender pan o comida los fines de semana"

El panorama de la educación boliviana ha tenido considerables avances a nivel de aumento de la escolarización y alfabetización, y a la vez persisten los desafíos en la permanencia y conclusión del año escolar, así como en la calidad de la alfabetización, los retos trasversales de interculturalidad en el sistema educativo y enseñanza de la lectura persisten en un 40\% (INE, 2016), esto en zonas urbanas. En zonas rurales persiste el desafío de género que se cruza con interculturalidad, hay familias que no envían a sus niñas al colegio porque consideran que su rol social es en casa, en los quehaceres de la granja o, en general, no consideran importante escolarizar a sus hijos porque su cosmovisión de la vida está arraigada en otros procesos de aprendizaje y saberes ancestrales, así también persiste la creencia de que la lectura altera la paz en el hogar y desordena la cabeza de las mujeres, por lo tanto no las envían al colegio, esto sobre todo en los territorios indígenas de pueblos originarios de la selva amazónica y del altiplano boliviano. 
El programa que más transformaciones ha dado a la educación boliviana (Cubas, 2006) es el bono escolar Juancito Pinto y que, sin embargo, es insuficiente, pero está avalado por la ley de educación. Ambas han sido las decisiones más significativas en los últimos 15 años, no obstante, no llegan consolidarse como políticas públicas plenas. Los objetivos específicos del programa bono escolar Juancito Pinto son: a) incrementar la matrícula escolar, disminuyendo la discriminación por clase social y etnia, b) reducir la deserción escolar y aumentar el ingreso de niñas y mujeres al sistema y c) aliviar la pobreza mediante la disminución de la trasmisión intergeneracional de la pobreza. Para ello, el objetivo inmediato del bono es aliviar los costos indirectos de la educación pública, tales como el transporte y los útiles escolares. Se trata de una transferencia monetaria condicionada destinada para que los niños y niñas cursen los ocho años del sistema educativo; seis de ellos correspondientes a nivel de primaria y los dos primeros cursos de secundaria de las unidades educativas fiscales (estatales) de todo el país, beneficiando a 1.925 .000 estudiantes en el año 2011 (INE, UDAPE). El monto del Bono es de Bs 200 (25 dólares) ${ }^{4}$ por estudiante, que son entregados a sus destinatarios una vez al año, en el mes de diciembre, bajo la dirección del Ministerio de Educación (Estado Plurinacional de Bolivia, 2006; UDAPE5 5 , 2011). Sin embargo, este monto no alcanza ni para comprar los textos escolares del año y es ínfimo en relación con las necesidades educativas y materiales del estudiante y sus familias.

Otro dato relevante, emitido por la Unidad de Análisis de Políticas Sociales del PNUD (2010), que permite dimensionar la educación y la lectura como factores del desarrollo humano y de un país, es que, en el estado plurinacional de Bolivia, los niños, niñas y adolescentes son más pobres que los adultos, y dentro de la niñez,

${ }^{4}$ Respecto a la significación del monto en la realidad boliviana, cabe señalar que el ingreso laboral promedio anual era en 2010 de 1632 dólares. El ingreso mensual de los ocupados en el año 2010 ( $2^{\circ}$ trimestre) era de 931 Bs (136 dólares) según la Encuesta de Empleo (1190 Bs para los varones y 631 para las mujeres). Fuente: INE http://www.ine.gob. bo/indice/EstadisticaSocial.aspx? codigo=30409.

${ }^{5}$ Unidad de Análisis de Políticas Sociales y Económicas del Ministerio de Planificación (significado de la sigla UDAPE). 
los más pobres son los menores de cuatro años (que experimentan malnutrición, la cual afecta enormemente sus procesos cognitivos al momento de ser escolarizados) con una marcada diferencia en las áreas urbanas y rurales. Esto considerando las especificidades de la pobreza infantil desde un enfoque de derechos, al que se corresponde una metodología específica de medición multidimensional de la pobreza, que además del nivel de gasto o consumo de los hogares considera variables como nutrición adecuada, salud, agua potable, servicios básicos, vivienda, educación y acceso a la información (aquí la lectura como competencia plena se vuelve un factor eje del desarrollo humano y marcador del índice de pobreza del país).

Lo afirmado en el párrafo anterior se sostiene con los indicadores sobre la pobreza infantil extrema, que alcanza en el país un $48,6 \%$, mientras que la pobreza total un $77,2 \%$ en el año 2007 , que hasta el 2014 han variado hacia arriba en un 3\%. En las áreas rurales las cifras llegan a un alarmante 78,2\% y 99\% respectivamente. Estos datos ubican al Estado Plurinacional de Bolivia como el país con mayor pobreza extrema infantil de América Latina y como uno de los que tiene mayor pobreza infantil total (CEPAL-UNICEF, 2010). Los datos denotan que ni la Ley de Educación 070, ni el bono escolar Juancito Pinto han abordado la educación parvularia, primaria y secundaria, ni resuelto las carencias del sistema educativo público en estos niveles, que además están atravesados por la salud, nutrición y cuidado materno-infantil, embarazo adolescente, falta de formación especial de profesoras para esta edad de 0 a 5 años $y$ expertos en lectura y escritura con enfoque en competencias textuales: crítica, reflexión e interpretación.

Además, persiste la trasmisión intergeneracional de las oportunidades educativas, que se expresa en las dificultades de acceso y conclusión de la educación secundaria y universitaria, sobre todo en las mujeres de zonas rurales y también de zonas urbanas periféricas. En este contexto, muchos países de América Latina han implementado programas de transferencias monetarias condicionados y dirigidos a la niñez y adolescencia, similares al bono escolar Juancito Pinto en Bolivia, insertados en sus matrices de protección social con las finalidades de disminuir la pobreza y mejorar el acceso a la 
salud y nutrición o la asistencia (aunque esto no asegura la permanencia) de niños, niñas y adolescentes en los centros educativos, así como de mejorar el nivel de ingresos y consumo de los hogares.

El programa bono Juancito Pinto tiene sus antecedentes directos en el programa implementado en la ciudad de El Alto, dado que, durante los años 2003 a 2005 el Gobierno Municipal de El Alto (localizado en el Departamento de La Paz) implementó el Bono Esperan$\mathrm{za}$, que consistía en transferir Bs. 200 (aproximadamente US\$. 28 dólares anuales) a los niños de este municipio, tenía la modalidad condicionada y era de carácter universal. Su objetivo era disminuir la deserción escolar ${ }^{6}$. Este bono a partir del año 2006 se convirtió en el bono Juancito Pinto a nivel nacional, y fue aprobado mediante el Decreto Supremo No. 28899, según este decreto, este es un programa de transferencia monetaria cuyos objetivos globales apuntan al desarrollo humano?.

A continuación, una tabla de elaboración propia que expresa las características socioeconómicas de la población objetiva del bono Juancito Pinto (los que asisten al colegio y trabajan, no asisten, asisten y no trabajan). Esta tabla permite conocer panorámicamente el contexto de los potenciales lectores del sistema educativo boliviano.

\begin{tabular}{lcccc}
\hline & No asiste & $\begin{array}{c}\text { Asiste y } \\
\text { trabaja } \\
\text { B }\end{array}$ & $\begin{array}{c}\text { Asiste y } \\
\text { no trabaja } \\
\text { C }\end{array}$ & $\begin{array}{c}\text { Total, } \\
\text { población } \\
\text { A+B+C }\end{array}$ \\
\hline Residencia en área rural (\%) & 64.89 & 32.66 & 42.35 & 44.29 \\
\hline $\begin{array}{l}\text { Residencia en ciudad } \\
\text { intermedia (\%) }\end{array}$ & 13.66 & 30.69 & 21.55 & 20.96 \\
\hline $\begin{array}{l}\text { Residencia en ciudad } \\
\text { capital (\%) }\end{array}$ & 21.45 & 36.55 & 36.10 & 34.75 \\
\hline $\begin{array}{l}\text { Origen indígena (\%) } \\
\text { Mujer (\%) }\end{array}$ & 55.16 & 62.79 & 56.39 & 56.37 \\
\hline $\begin{array}{l}\text { Escolaridad promedio del } \\
\text { jefe de hogar (años) }\end{array}$ & 56.43 & 27.50 & 48.81 & 49.19 \\
\hline
\end{tabular}

${ }^{6}$ Diseño de una Red Integral de Protección Social en Bolivia. Gabriel Loza - UDAPE (2005)

${ }^{7}$ CEPAL - Flavia Marco Navarro (2012) en base a UDAPE, 2011 


\begin{tabular}{lcccc}
\hline $\begin{array}{l}\text { Ingreso mensual } \\
\text { per cápita (Bs) }\end{array}$ & 174.08 & 351.49 & 351.36 & 335.42 \\
\hline Indigente (\%) & 64.43 & 31.79 & 48.98 & 50.12 \\
\hline Pobre (\%) & 87.42 & 54.27 & 70.89 & 72.14 \\
\hline
\end{tabular}

Fuente: Elaboración propia a partir de datos del Instituto Nacional de Estadística (INE, 2013) y la Encuesta de Hogares, tomados el año 2005, por el mismo Ministerio de Educación y el de Planificación, para el diseño del programa.

Como se observa en los tres grupos considerados (A, B, C), la población que no asiste al colegio se concentra en el área rural, un 64.9\% de la población que no asiste a ningún centro de primaria reside en el sector rural. Lo contrario ocurre en las otras dos categorías donde se evidencia una mayor participación de la población urbana. La población indígena es mayoritaria en todas las categorías, y particularmente en el grupo de personas que trabaja y estudia. En promedio, son las mujeres las que predominan en el grupo de personas que no estudian y no saben leer, mientras que los varones tienen una mayor presencia en los otros dos grupos. Se confirma la importancia del capital humano de la familia, ya que la escolaridad de los jefes y jefas de hogar es creciente en relación a la importancia que se le da a la educación. Por ejemplo, mientras el promedio de escolaridad en el primer grupo -los que no estudian- es de 4,2 años, en el grupo de individuos que sólo estudia, la escolaridad del jefe llega a 7,1 años.

Estos datos evidencian los elevados niveles de pobreza de Bolivia y la relación directa entre pobreza, educación y lectura; pobreza, alfabetización y género; pobreza e interculturalidad (la lectura en estos contextos), ya que en todas las categorías la proporción de pobres supera el 54\%. Se evidencia también que el grupo de individuos que no estudia proviene de hogares con niveles de ingresos per cápita más bajos que los observados en los otros grupos, lo que se refleja en la alta proporción de indigentes y pobres que este grupo incluye.

El programa del bono escolar Juancito Pinto expresa complementariedad con otras iniciativas nacionales, dado que responde a un proceso histórico y de interés en los distintos gobiernos de la historia 
de Bolivia (aunque siguen sin resolverse los problemas estructurales de la educación, la ausencia de políticas de lectura y la pobreza). Además, esta preocupación se ha profundizado con el cumplimiento del segundo objetivo del milenio que es alcanzar la educación primaria universal. Bajo esta lógica el 2006 se implementa a nivel nacional el bono Juancito Pinto, para incentivar la permanencia de los niños y niñas de primaria en escuelas públicas, y de manera articulada se ejecuta el Programa Nacional de Alfabetización -"Yo sí puedo"- para erradicar el analfabetismo absoluto y funcional de la población boliviana, 1.200 .000 personas -en el 2006- según el Ministerio de Educación (CEPAL, 2020), pero en ningún caso se establece una política pública plurinacional de lectura que resuelva sostenidamente, con recursos anuales, los problemas mencionados, ni siquiera después de la promulgación de la Ley del Libro y la Lectura "Oscar Alfaro" (2013) se toman decisiones al respecto.

En relación con decisiones complementarias a este programa, se han visto intervenciones orientadas a incrementar la oferta (construcción de escuelas), subvencionar la demanda (desayuno escolar, transporte escolar, albergues escolares, etc.) y enfrentar problemáticas específicas (reinserción educativa de niños y adolescentes trabajadores), campañas de recolección de libros, construcción comunitaria de bibliotecas, según declaraciones del viceministro de educación. En este contexto, las últimas décadas han mostrado el surgimiento de "una nueva generación de programas sociales" (CEPAL, 2020) que en Bolivia ha dado resultados positivos a corto plazo, pero que no han resueltos los desafíos estructurales que hay en la educación boliviana en relación con la calidad y las políticas de lectura.

Otro aspecto en torno al bono escolar Juancito Pinto y la educación boliviana es el de la falta de ejercicio de los derechos en la niñez y adolescencia, que está atravesado por los derechos de las niñas de los pueblos originarios, lo cual al mismo tempo está vinculado con la pobreza, y a su vez provoca el aumento del trabajo infantil; por lo mismo, este es más alto entre la población rural e indígena, como lo vimos en la tabla anterior. De hecho, el ejercicio deficitario de derechos en la infancia, la niñez y la adolescencia es parte de la pobreza; 
a su vez, la insuficiencia de ingresos en jefes o jefas de hogares; otra de las dimensiones de la pobreza es la que motiva a niños, niñas y adolescentes a salir del hogar en busca de dinero, conformando un círculo vicioso.

El trabajo infantil en Bolivia carece de regulación y fiscalización, dificultando el acceso a la educación, la permanencia y desempeño de la niñez y adolescencia en las escuelas y además afecta otros derechos consagrados legalmente. Es el caso de los derechos a la libertad, a la educación, integridad física, sexual y moral, a vivir con la familia, al deporte y al juego, entre otros (Ley de Educación 070, Estado Plurinacional de Bolivia, 2010). Complementando este argumento el Banco Interamericano de Desarrollo, (2007) alude a los bajos niveles educativos en la región como el principal obstáculo para lograr una mayor productividad del trabajo y, consecuentemente, mayores indicadores de crecimiento económico y de reducción de la pobreza, fortalecimiento de la democracia, disminución de autoritarismos, premisa que estaría sustentada en la evidencia empírica.

En el mismo sentido, el Índice Municipal de Desarrollo de la Infancia, Niñez y Adolescencia ${ }^{8}$ (IDINA) y datos del gobierno municipal de los departamentos de Santa Cruz, La Paz, Cochabamba y Beni, muestra que los niños y niñas menores de 6 años son quienes tienen un menor ejercicio de sus derechos de acceso al libro y la lectura, de profesores con ítems asegurados, y que al nivel inicial correspondía una proporción menor al 1\%, ínfima inversión en educación (UDAPE-UNICEF, 2008 y Defensoría de la Niñez y Adolescencia del Gobierno Autónomo Municipal de Santa Cruz, 2009). En el promedio nacional el IDINA muestra un moderado cumplimiento en

${ }^{8}$ El Índice Municipal de Desarrollo de la Infancia, Niñez y Adolescencia (IDINA) es un instrumento de medición del grado de cumplimiento de derechos de los niños, niñas y adolescentes en el país, construido a través de la comparación relativa de indicadores relacionados con cuatro dimensiones de análisis: salud, educación, capacidad económica, habitabilidad, derecho a la identidad y protección contra los riesgos de explotación económica y maltrato. En el 2005 la inversión en educación inicial fue de 15,5 millones de dólares, mientras que en secundaria de 80,5 y en primaria de 288 millones. Considerando el presupuesto total en educación, el nivel inicial corresponde al 0,8 y 0,5\% en las áreas urbanas y rurales respectivamente (UDAPE, 2008). 
el ejercicio de los derechos de la niñez y la adolescencia con un valor de 0,62 sobre un máximo de 1 (UDAPE-UNICEF, 2012), que tampoco resuelve los niveles de educación de calidad. Respecto a los objetivos del programa, vemos que el único logro se experimenta en el nivel de disminución de la tasa de abandono escolar en los niveles inicial y primario, que es de $5 \%$.

Al respecto, Aguilar (2014) explica que:

"Un beneficio tangencial a los objetivos, el derecho a la identidad también representa avances con un $94 \%$ de los niños, niñas y adolescentes con certificado de nacimiento. Este progreso en el derecho a la identidad está relacionado con la gratuidad del primer certificado de nacimiento, que puede obtenerse de esta manera y sin trámites adicionales hasta los doce años de edad, lo cual antes del gobierno de Evo Morales tenía un costo de 50 Bs. Sin embargo, siguen los desafíos a nivel burocrático que dificultan el ejercicio del derecho a la identidad de niños, niñas y adolescentes y por lo tanto el cobro del bono escolar; sobre todo relacionados con la obtención de la cédula de identificación, ya que las oficinas de Identificación no están en las zonas rurales de todo el pais, y muchas familias no tienen dinero para viajar con sus hijos hasta la ciudad, esta situación dificulta o impide el cobro del bono; faltan recursos y tecnología para resolverlo."

En relación con la educación, aparecen también diferencias de género que se dibujan como poco significativas, sin embargo, los promedios nacionales podrían estar escondiendo diferencias de género a nivel local y en regiones alejadas de las ciudades capitales (en poblaciones originarias como la ayoreode, guaraní, mojeña, etc.).

Es relevante mencionar que junto a este programa se ha establecido como política pública educativa, además del bono Juancito Pinto, una serie de acciones en el marco legal: La nueva Constitución Política del Estado menciona en su artículo 17 que "toda persona tiene derecho a recibir educación en todos los niveles, sin discriminación por género u origen social, étnico”. La Ley de Educación "Elizardo Pérez y Avelino Siñani" que es llamada un instrumento de la revolución educativa, ya que dará una de manera universal, productiva, gratuita, integral e intercultural, sin discriminación, una "educación para todas y todos con calidad para vivir bien". 
Por su parte, a nivel institucional se realizaron en los últimos 5 años programas de propaganda política que figuraban como políticas educativas, entre ellos el denominado programa de alfabetización "Yo sí puedo", "Yo sí puedo seguir", programa de profesionalización de maestros y maestras, programa de formación complementario para maestros y maestras, acto simulación de entrega de más de 100.000 computadoras a los estudiantes de todo el país y la implementación del RUE (Registro Único de Estudiantes). Todo ello más con fines estadísticos, para justificar que están trabajando por el desarrollo del país, tomar las fotos de rigor y generar informes, pues hay antecedentes de que los estudiantes no pudieron acceder a las 100.000 computadoras de manera libre y única.

En relación al bono Juancito Pinto que sigue en vigencia, todavía no se puede visualizar su efectividad porque muchos estudiantes siguen abandonando a mitad de año el colegio, retoman un mes antes de las evaluaciones, cobran el bono, se inscriben para el siguiente año escolar, y así, ante la ausencia de monitoreo del programa se genera una lógica asistencialista y un programa que se auto engaña, a cada beneficiario y a la población.

Otro dato al respecto es que estas acciones y programas, para supuestamente reducir las desigualdades, la pobreza y aumentar la escolarización, etc. no han sido suficientes, ya que el 27\% (INE:2009) de los niños no se matricula en los establecimientos educativos, según la Encuesta de Trabajo Infantil del año 2008; 27\% de los niños entre 5 y 17 años trabajan o realizan alguna actividad económica en los negocios familiares, granjas, etc. o en las calles, para llevar dinero a sus hogares, no están escolarizados o si lo están, no asisten y dado que la nueva Ley de Educación 070 prohíbe reprobar a un estudiante (ello es discriminación en la nueva CPE del Estado Plurinacional de Bolivia), estos muchas veces se inscriben, cobran su bono y el resto del año no asisten a clases y se dedican a trabajar.

En este sentido de género y desigualdad social, será necesario que las políticas públicas tengan una focalización necesaria para homogeneizar los beneficios de las mismas entre todos los niños, en 
especial en los más vulnerables, ya que las necesidades de los niños de 0 a 5 años son más profundas, dependiendo del tipo y nivel de vulnerabilidad en la que se encuentran (rural o urbano) ${ }^{9}$.

En función a estas reflexiones y hallazgos, para posible tesis o investigación de campo es urgente rediseñar las políticas educativas y culturales, bajo un enfoque de género y diversidad cultural. El diseño analizado anteriormente sobre la política del Bono Juancito Pinto no permite realizar una evaluación de impacto para mejorar el monitoreo y evaluar los resultados sobre la variable de interés en esta política del Bono Juancito Pinto (tasa de matriculación, calidad de la alfabetización y permanencia escolar) y, por consiguiente, esta política educativa está limitada a aumentar la estadística en favor de una gestión de gobierno, la cual no impacta en los problemas estructurales de la educación, la lectura, y los derechos de la niñez y juventud en Bolivia. Si bien no se habla de permanencia escolar en los objetivos, es una variable que debió considerarse en el diseño de programas y políticas públicas, puesto que, como indicó el ex presidente de la Federación Urbana de Maestros en Santa Cruz de la Sierra, Bolivia, en entrevista, el profesor José Miguel Abasto (2020): “(..) muchos niños son matriculados, aparecen los números en las estadisticas de alfabetización y escolarización, en aumento, pero no permanecen estudiando, dejan la escuela a mitad de año, siguen trabajando con sus padres, en la calle, en sus granjas, van a rendir exámenes que reprueban, pero la Ley de Educación prohíbe reprobar a los estudiantes, entonces no ven la obligatoriedad de permanencia $y$ concluir el año escolar y concluyen con notas bajas, cobran su bono, llenan la estadística y vuelven a su campo de trabajo y subsistencia. Entre el 2006 y el 2012 el programa escolar y la misma ley de educación boliviana, han tenido algunas transformaciones, inicialmente -en el 2006- la población beneficiaria eran todos los niños y niñas que asistian a escuelas fiscales y cursaron los primeros cinco años

${ }^{9}$ El Plan Nacional de Desarrollo menciona que la "Estrategia de Generación de Oportunidades y Activos Sociales está dirigida a la creación de activos sociales para las poblaciones con mayores niveles de pobreza" dentro de esta estrategia se encontraría el programa Bono Juancito Pinto. 
de primaria; para el 2007 la población objetivo aumentó tomando en cuenta a los niños y niñas de sexto de primaria e incluyó a todos los asistentes a los centros de educación especial ${ }^{10}$ y a los adolescentes de la educación juvenil alternativa ${ }^{11}$, el 2008 se extiende a los niños y niñas de séptimo y octavo de primaria $y$, finalmente el año 2012 se amplia hasta tercero de secundaria, es decir que cubriria 11 de los 12 años que se necesitan para salir bachiller (requisito para ingresar a la educación superior o universitaria). Siguen los problemas estructurales, de falta de establecimientos, recursos didácticos para los colegios, libros, computadores, actualización docente útily no sobre ideologias politicas, faltan también items para los colegas profesores, (...)"

Explicitando la intervención del profesor José Miguel Abasto, se aclara que: Para que el niño o la niña puedan recibir el bono Juancito Pinto, y anexarse a los beneficios de la Ley 070 de Educación, es necesario que se hayan matriculado y hayan tenido una asistencia regular durante la gestión escolar (no menos de 80\% de asistencia), aunque estas pautas no son respetadas en los hechos, ésta es certificada por un profesor, el Director de la Escuela y/o la Junta Escolar, asimismo el beneficiario debe contar -de manera obligatoria-con el Registro Único de Estudiantes ${ }^{12}$ (Ministerio de Educación, 2012). El límite de edad para recibir el mismo es de 18 años de edad en el caso de la educación formal y la educación juvenil alternativa, para el caso de los niños en educación especial no se tiene límite de edad ${ }^{13}$.

10 "Son los estudiantes que se encuentran en una situación especial debido a sus características bio- psico- sociales, y por tanto necesitan una atención especial y el uso de técnicas y procedimientos particulares”. (Yáñez, 2012 y Ley de educación Elizardo Pérez y Avelino Siñani).

11 "Son los adolescentes que no pueden continuar con sus estudios en el área de la educación formal y asisten a los Centros de Educación Juvenil Alternativa que ofrece varias modalidades (escolarizadas, no escolarizadas, presenciales, a distancia)”. (Yáñez 2012 y Ley de educación Elizardo Pérez y Avelino Siñani).

${ }^{12}$ Explica el Ministerio de Educación y deportes, que el objetivo del RUE es asentar la información de los estudiantes en un sistema informático, en la escuela, en el distrito, en la Dirección Departamental de Educación y en el Ministerio de Educación, para que esté al alcance de los propios estudiantes y padres de familia y para una mejor planificación educativa.

13 Yáñez (2012). 


\section{Conclusiones y discusión}

De acuerdo a la pregunta principal de investigación: ¿Cuál es la noción de interculturalidad educativa y el valor que asigna el Estado Plurinacional de Bolivia a la lectura, la memoria oral, las bibliotecas y la educación?, se observa que el valor lo otorgan los profesores desde sus prácticas pedagógicas y didácticas en torno a la lectura, desde una lógica de didácticas para la lectura en contextos de alta diversidad cultural.

No hay valoración didáctica, pedagógica, dialógica intercultural por parte del estado, más allá del gobierno vigente, lo que existe es una politización de los significados, una producción de leyes que respalden la estadística intencionalmente generada para favorecer una gestión y otra en los discursos políticos. Por otra parte, se evidencia la desarticulación entre la legislación, las políticas educativas y culturales y la realidad expresada por los profesores entrevistados. Las brechas educativas y de políticas culturales que favorezcan la lectura y la pacificación del país, son enormes, a nivel nacional e internacional.

El porcentaje de alfabetización representada en la estadística boliviana en aumento, se contradice con las entrevistas realizadas a profesores y los informes generados anualmente, debido a que no se logra salir del umbral lector para alcanzar niveles de competencias textuales, que permitan alcanzar estudios superiores, sin deserción o dilatación de la etapa universitaria en pre grado.

Lo anterior se explica como una consecuencia en el modo de administración de las políticas educativas y culturales del país, que no descentraliza recursos para la actualización pedagógica, didáctica y tecnológica para el profesorado escolar y universitario, así mismo se carece de la implementación de bibliotecas escolares y descentralización de fondos para la actualización bibliográfica y capacitación de capital humano en el país.

Cabe destacar que el asunto de la enseñanza de una lengua originaria en el sistema educativo, impacta el proceso de identidad y resguardo del patrimonio cultural inmaterial de un país, en el caso boliviano, se incorporan solo tres lenguas y se desmedran las otras 
33 variantes socioculturales e identitarias, no se realizan estudios sistemáticos sobre la memoria y tradición oral, tampoco se valoran las prácticas pedagógicas, implementadas por los profesores en los distintos lugares del país.

En relación a las lenguas y el patrimonio cultural inmaterial del país, el Viceministerio de Interculturalidad que dependía del Ministerio de Culturas, delimitó su campo de acción a los mandatos constitucionales político-ideológicos de la Ley Marco de Autonomías Indígenas, pero no se valoró el patrimonio oral, asignando presupuestos para profesores de las zonas rurales de tierras bajas de Bolivia, por lo que persiste el problema de la ausencia de salarios en las zonas periféricas de las ciudades capitales y en las zonas rurales.

En relación al sistema político y administrativo de la educación, no se asignan fondos para investigaciones sistemáticas en lengua, cultura y patrimonio cultural inmaterial de Bolivia. Los 4 niveles de administración pública: nacional, departamental, municipal y originario campesino no incorporan estos elementos como prioridad en sus presupuestos y proyectos de desarrollo humano, lo cual deja en evidencia el absoluto desinterés por resguardar la diversidad cultural y divulgarla. De ello se harán cargo los jóvenes profesores con capacidad organizativa, en el caso de los chiquitanos, y los mismos guaraníes y moxeños, por mencionar algunos.

Más allá de la crítica de los documentos, también se critica el status social de los documentos. Este estudio, en particular, espera ser un aporte al mejoramiento de las políticas públicas educativas y de lectura en Bolivia, al finalizar el mismo, y habiendo abordado la lectura y lo intercultural en la educación como punto de partida, se obtuvo que los significados de interculturalidad, memoria oral, educación y lectura en el marco jurídico se definen como elementos políticos para conseguir votos y fondos internacionales y que fueron ejecutados sin transparencia ni participación de los profesores y actores culturales del país.

Las entrevistas a los profesores y el análisis en detalle de las categorías, subcategorías y categorías emergentes, devela que los profesores son conscientes de que la producción de leyes generada durante el gobierno del Movimiento al Socialismo (MAS) fue parte 
del ensamblaje discursivo que hizo de sus necesidades educativas, en torno a la lectura y el patrimonio cultural, su bandera política para conseguir votos y gobernar por muchos años.

Las entrevistas y análisis documentales de la legislación boliviana, en relación a la educación, la cultura y la lectura, dan cuenta de las inequidades y brechas sociales, educativas a nivel de género, a nivel de participación de la diversidad cultural del país y a nivel de acceso a la calidad educativa, tecnologías y libros.

Esta realidad develada desde el análisis documental y las entrevistas a profesores, que forman parte de la federación urbana y rural de maestros en Bolivia, evidencia la urgencia de un plan de gobierno en favor de la educación, la diversidad cultural y la lectura, la participación de los profesores y estudiantes en la elaboración de proyectos, y acciones en relación a sus necesidades de aprendizaje para resolver las brechas cognitivas y de desarrollo educativo persistentes hasta la actualidad.

En el análisis se afirma la monopolización del poder político a punta de producción de leyes y la existencia de un Ministerio de Culturas y un Viceministerio de Interculturalidad para fomentar la creación de leyes y documentos que sustentan el monopolio político de un partido en relación a su propia ideología, que fomenta la violencia interétnica, con discursos de interculturalidad y hermandad, y en contradicción a ello no se ven políticas públicas a largo plazo, no se descentraliza ni genera participación plena de las distintas zonas del país en los procesos de elaboración de contenido y currículo educativo en torno al patrimonio cultural inmaterial del país.

Por otra parte, se evidencia la ausencia de investigación en lenguas y tradición oral del oriente boliviano, para su debida enseñanza y transferencia cultural en el marco de la diversidad cultural y las necesidades globales a escala humana, en las cuales están insertos los niños y jóvenes de Bolivia. Se corrobora el supuesto de que la interculturalidad se politiza en favor de intereses partidarios y resulta en falacia a nivel de procesos educativos y culturales en uno de los países más diversos del mundo.

Es todavía un reto para Bolivia asumir un modelo de desarrollo basado en la educación y la diversidad cultural, con enfoques 
pedagógicos y didácticos que incorporen la riqueza de las prácticas docentes que están en la cultura de Bolivia, pero que no son consideradas en el sistema. Se necesita establecer una política pública educativa que potencie y haga sostenible los alcances del modelo pedagógico que cada cultura tiene, y está directamente asociado con sus prácticas de interacción con la naturaleza y la vida.

Mientras no se asuman modelos pedagógicos desde las prácticas reconocidas y el respeto a los profesores que están hace más de 40 años ejerciendo la docencia y resistiendo con honor la brutalidad del centralismo administrativo, se seguirán repitiendo practicas desarrollistas extractivistas de anteriores procesos históricos (como la revolución del 1952), y se seguirá siendo cómplice de la violencia sistemática que atraviesa la vida de los profesores ante el abandono y la ausencia de recursos mínimos para ejercer sus oficios con más tranquilidad.

Las autoridades tienen la responsabilidad de generar una legislación boliviana educativa descentralizada en relación a la administración de nuestros recursos, que priorice el desarrollo humano, honre la vida de los profesores en cada región del país, respetando sus prácticas pedagógicas y didácticAS, RESGUARdando sus variantes socioculturales y lingüísticas, como parte del patrimonio cultural, natural e identitaria de cada zona.

Es urgente superar los caudillismos y colonialismos internos que subyacen en el sistema de gobierno y el sistema legislativo nacional, que generan las precariedades evidenciadas en este estudio en la ausencia de políticas educativas sostenibles en favor de la población. Queda claro que gestionar el patrimonio cultural y natural, desde la educación y actualización administrativa, legislativa de todo el sistema educativo boliviano es una prioridad, para lograr la cohesión social y el diálogo intercultural para evitar conflictos interétnicos, a raíz de los programas políticos vigentes. 


\section{Referencias:}

Ábrego y Quiróz (2020). Historia de la Unión de Grupos Culturales. Santa Cruz de la Sierra: Mesa Río.

Albó, X. (2009). Autonomías indígenas en la realidad boliviana y su nueva constitución. La Paz: Vicepresidencia del Estado Plurinacional.

Arias V. y Giraldo C. (2011). El rigor científico en la investigación cualitativa. Revista Investigación y educación en enfermería, 3, 500-514.

Aguilar H. (2014), Evaluación del Bono Juancito Pinto. Revista de análisis, 21. 45-57

CERLALC (2020). Nueva agenda por el libro y la lectura: recomendaciones para políticas públicas en Iberoamérica. Recuperado de: https://cerlalc. org/wp-content/uploads/publicaciones/olb/PUBLICACIONES_OLB_Nueva-agenda-por-el-libro-y-la-lectura_v1_011013.pdf

CEPAL (2020). Distribución espacial y urbana de la población de América Latina y el Caribe. Recuperado de: https://bit.ly/3kuOuLv.

Cubas, R. P. (2006). Bolivia: Movimientos sociales, Nacionalización y Asamblea Constituyente. Observatorio Social de América Latina, CLACSO, 19, 55-64.

Instituto Nacional de Estadística de Bolivia, INE (2010). Encuesta Nacional de Empleo. Recuperado de: http://www.ine.gob.bo/indice/EstadisticaSocial.aspx? codigo $=30409$

Ley 366, del Libro y la Lectura Oscar Alfaro. Gaceta Oficial de Bolivia. 30 de abril de 2013

Ley 070 de Educación Avelino Siñani-Elizardo Pérez. Gaceta Oficial de Bolivia.20 diciembre de 2010.

Ley 31 Marco de Autonomías y Descentralización Andrés Ibáñez. Gaceta Oficial de Bolivia. 19 de julio de 2010.

Loza, G. (2005) Diseño de una Red Integral de Protección Social en Bolivia. Revista de Análisis Económico UDAPE, 22, 164- 196.

Ministerio de Autonomías (2012). Clasificador competencial: Autonomías Bolivia. Serie 2, autonomías para la gente. Recuperado de: http://vpc. planificacion.gob.bo/uploads/recursos/Clasificadorcompetencial.pdf

Ministerio de Educación (2016). Comunicación y Lenguajes: Lenguas Castellana y Originaria - Sistemas de Comunicación y de Lenguajes de Nuestros Pueblos y Naciones” Cuadernos de Formación Continua. La Paz: Equipo PROFOCOM. 
Ministerio de Educación (2017). El Desarrollo de la Lengua Oral y Escrita en Primaria Comunitaria Vocacional. Recuperado de: https://docplayer. es/64027229-El-desarrollo-de-la-lengua-oral-y-escrita-en-primaria-comunitaria-vocacional.html

Molina, C. (2018). La nación de los indios chiquitos. Cepad Ediciones.

PNUD (Programa de las Naciones Unidas para el Desarrollo Humano). (2016). Informe sobre el desarrollo humano. Washington: Communications Development Incorporated,

Peña, P. (2020). La permanente construcción de lo cruceño. La Paz: PIEB.

UNESCO (2003). Convención para la salvaguardia del patrimonio cultural inmaterial. Recuperado de: http://portal.unesco.org/es/ev.php-URL_ ID=17716\&URL_DO=DO_TOPIC\&URL_SECTION=201.html

Urenda, J. (2018). Autonomias centralizadas en Bolivia. La Paz: Ediciones El País.

Vaca, C. (2018). El metarrelato autonomista de Santa Cruz de la Sierra. (Tesis para Maestría en Ética Social y Desarrollo). Universidad Alberto Hurtado, Santiago.

Valenzuela, E., Cienfuegos, I. y Henríquez, O. (2019). Los tres modelos de descentralización subnacional de Sud América. Revista RAP, 53, 1-27.

Valenzuela, E. y Vaca C. (2020). "Ciudades contrapeso al centralismo unitarista en Sudamérica: Santa Cruz, Guayaquil y Concepción”. Íconos. Revista de Ciencias Sociales, 68,171-189.

Yáñez, E. (2012). El impacto del bono Juancito Pinto. Un análisis a partir de microsimulaciones. LAJED, 17, 75-112.

Zavala, V. (2001). Oralidad y escritura en la educación bilingüe. (A propósito de interculturalidad). Lima: MED-Plancad-GTZ-KfW.

Recibido: 22.07.2020 Aceptado: 16.10.2020 